\title{
Cell Virtualization with Network Partition for Initial User Association in Software Defined Small-cell Networks
}

\author{
Guolin Sun ${ }^{1}$, Li Lu ${ }^{1}$, Daniel Ayepah-Mensah ${ }^{1}$, Xiufen Fang ${ }^{2}$, Wei Jiang ${ }^{3,4}$ \\ ${ }^{1}$ School of Computer Science and Engineering, University of Electronic Science and Technology of China \\ Chengdu, 611731, China \\ ${ }^{2}$ School of Mathematical Sciences, University of Electronic Science and Technology of China \\ Chengdu, Sichuan, 611731, P. R. China \\ ${ }^{3}$ German Research Center for Artificial Intelligence (DFKI GmbH), Kaiserslautern, Germany \\ ${ }^{4}$ Department of Electrical and Information Technology (EIT), Technische University (TU) Kaiserslautern, \\ Germany \\ [e-mail: guolin.sun@uestc.edu.cn] \\ *Corresponding author: Guolin Sun
}

Received December 19, 2016; revised May 21, 2017; revised January 10, 2018; accepted April 18, 2018; published October 31, 2018

\begin{abstract}
In recent years, dense small cell network has been deployed to address the challenge that has resulted from the unprecendented growth of mobile data traffic and users. It has proven to be a cost efficeient solution to offload traffic from macro-cells. Software defined heterogeneous wireless network can decouple the control plane from the data plane. The control signal goes through the macro-cell while the data traffic can be offloaded by small cells. In this paper, we propose a framework for cell virtualization and user association in order to satisfy versatile requirements of multiple tenants. In the proposed framework, we propose an interference graph partioning based virtual-cell association and customized physical-cell association for multi-homed users in a software defined small cell network. The proposed user association scheme includes 3 steps: initialization, virtual-cell association and physical-cell association. Simulation results show that the proposed virtual-cell association outperforms the other schemes. For physical-cell association, the results on resource utilization and user fairness are examined for mobile users and infrastructure providers.
\end{abstract}

Keywords: User association; Cell virtualization; Software defined network; Network partition; 


\section{Introduction}

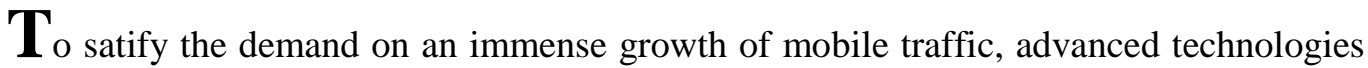
such as multi-homed equipment and multi-path transmission are investigated by both academia and industry [1][2]. However, user association problem, namely how users select its serving Access Point (AP), are complicated [3]. Finding an efficient way of user association is the key to achieve the best resource utilization. However, user association management in a multitude of users and APs is not an easy job. The general user association plays a pivotal role in enhancing network performance. In general, each AP in a Wi-Fi network periodically broadcasts the beacon frames to announce its presence. When a user equipment is turned on or reconnect to a network, it scans those signals to measure their received strengths and therefore decides to connect to the AP with the strongest Received Signal Strength Indicator (RSSI). In the system with uneven user distribution, all users prefer to connect to a powerful AP regardless of other factors. This pure RSSI based user association results in some APs being overloaded, while others are idle. This leads to the mediocre resource utilization as it decreases the Quality of Service (QoS) for users.

On the other hand, because of diverse application requirements on network protocol design, different tenants prefer to define their own user association schemes for different optimization objectives. The introduction of Software Defined Network (SDN) reduces the complexity of flow control by decoupling the control plane from the data plane [4]. In the literatures,various software defined user association schemes have been proposed[3]. Notably, the programmable WLANs with Odin [5] and Cloud-MAC [6] are discussed. They propose the control logic to manage a crowd of resources in a software defined Wi-Fi network. Meanwhile, network function virtualization will play a pivotal role in the future evolution of information and communication technology. It can reduce the CapEx and OpEx by creating virtualized network slices to satisfy the diverse demands of mobile users [7]. Cell virtualization and user association have been introduced under the architecture of Cloud Radio Access Networks (Cloud-RAN) [8]. However, APs in a static cluster must share a common cloud of Base-Band Units (BBUs). Anyway, it has been noted that we require the appropriate schemes for user association for different services in virtualized radio access networks.

Large network scale leads to high computation complexity in Cloud-MAC and Cloud-RAN. Therefore, we propose a dynamic clustering scheme via graph partitioning and allow versatile user association via small cell virtualization in SDN. Particularly, the objective is to fairly balance the traffic load in a partitioned network within a system of virtualized cells and multi-interfaced users. In SDN-based heterogeneous cellular network, small cells are used to offload data traffic from Macro-cell in terms of physical Transmission Points (TPs). The concept of TP is introduced to differentiate APs that are used to offload traffic. AP has both control plane and data plane, but a TP is mostly responsible for data transfer. The potential application of TP includes versatile relay system [9][10] and secondary eNodeB selection in the dual-connectivity mode of 5G system[11]. The main contributions of this paper can be summarized as follows:

- A framework of versatile association manager based on cell virtualization is built for a software defined small cell network. It achieves the isolation among virtual cells in a self-organized manner in terms of Virtual Transmission Points (VTPs). It allows customized physical-cell association for different tenants.

- An interference graph partitioning algorithm has been developed for virtual-cells 
association based on Kernighan-Lin Algorithm (KLA). The clustering scheme on the interference graph is to swap TPs to maximize the utility function. The utility function is defined as the difference of average Signal to Interference plus Noise Ratio (SINR) between intra-virtual-cell and inter-virtual-cell.

- Comprehensive simulations are done to show the effectiveness and performance of the proposed algorithm from the perspectives of virtual mobile network operators, physical network operators and users.

The rest of this paper is organized as follows: Section 2 summarizes the related works, while the proposed architecture and algorithms are presented in Section 3. The experiment results are given in Section 4 and finally, we conclude this paper in Section 5.

\section{Related Works}

In small cell networks, traffic load balancing is an essential task for network performance optimization and has been extensively studied [12]. In mobile networks, traffic load balancing is achieved by executing a user association process, in which mobile users are assigned to the APs. In [13], authors present an algorithm for the throughput maximization problem by selecting the active RRHs and selects a subset of active RRHs that maximize the system throughput under the front-haul constraint. Cell biasing algorithms are developed in [14] to balance traffic load and also enhance network QoS among macro-BSs and pico-BSs. The cell biasing algorithm performs user association based on the biased measured pilot signal strength and off-loads traffic from macro-BSs to pico-BSs. Authors proposed a dynamic user association algorithm in [15] to maximize the sum rate of network and adopted cell biasing to balance the traffic load among BSs. On the other hand, authors proposed an alpha-optimal user association algorithm to achieve flow-level load balancing under spatially heterogeneous traffic distribution [16]. The proposed algorithm may maximize different network utilities, e.g. the traffic latency and the network throughput, by properly setting the value of alpha. In [17], authors proposed a network latency-aware user association scheme to minimize the average traffic delivery latency for backhaul constrained small cells.

Energy consumption is one of the metrics to measure the efficient resource utilization. Thus, in [18], a user association in heterogeneous cellular networks was proposed to optimize energy utilization. Numerous valuable contributions have been published on energy efficient user association in HetNets. In [19], user association for the downlink of HetNets was optimized by maximizing the ratio between the total data rate of all users and the total energy consumption. The authors in [20] investigated how to maximize the energy efficiency defined as the ratio of the achieved throughput over the energy cost by optimizing the time allocation for the downlink and multi-user uplink traffics for the simultaneous wireless information and power transfer systems.

The outage coverage probability is used as the primary performance metric employed for user association analysis in conjunction with stochastic geometry. In particular, the authors of [21] and [22] modeled and analyzed the performance of max-RSS user association in K-tier downlink Het-Nets with the aid of stochastic geometry. The SINR model in [21] was improved in [22] in order to account for the activity factor of the co-existing heterogeneous BSs. It was shown that adding lightly-loaded femtocells and pico-cells to the network increases the overall coverage probability.

In [5], authors proposed a prototype of SDN framework called Odin for enterprise WLANs. Odin builds on a Light Virtual Access Point (LVAP) abstraction that virtualizes association 
state, and thus results in multiple virtual clients connected to a single physical AP. Authors introduced Cloud-MAC in [6] as a novel management architecture, in which APs just forward MAC frames. All other functionalities, such as the MAC data or management frames processing, are implemented in a cloud server. A technique that uses UE-centric metrics to partition the network into multiple overlapping VTP sets is introduced in [24], which also considers TP's load to calculate their bonding level. However, no study has been done on multi-homed users or on theoretical analysis in virtualized radio access network. Multi-layer virtualization schemes were presented as cell and user virtualization for ultra-dense network [24], where authors virtualized different network component to tackle the interference and mobility issues in dense network. Unfortunately, except for multi-layer virtualization, no appropriate scheme for user association is presented. To choose appropriate small cells for users, the user association scheme in cloud-RAN based small cell network with wireless virtualization was studied in [8]. To meet the diversity requirements from users and multiple tenants, network virtualization enables mobile service providers to program and deploy their own typical enterprise LAN services in an independent manner. In [27], a dynamic user clustering method has been investigated to minimize the intra-cluster interference. Contrary to the aforementioned association schemes, flexible user association in virtualized TP networks has to be done considering intra-cluster and inter-cluster signal-to-inteference.

\section{The Proposed Scheme}

\subsection{System architecture}

In this section, we present the system architecture followed by the problem addressed in the paper. Fig. 1 shows the proposed network architecture and signaling for the initial user association. As illustrated in Fig. 1, a heterogeneous small cell network is composed of an LTE Macro-cell and several TPs, such as Femtocells or relay nodes. This heterogeneous small cell network is programmable and managed by an SDN controller. Under this SDN architecture, decoupling of control plane and data plane is achieved. The control signaling goes through macro-cells, whilst data traffic is mostly offloaded to small cell TPs. The SDN controller manages the system and makes decision on user association.The physical resource request and allocation includes the process between the user and the TP without the controller. In this scenario, a set of multi-homed users, which are characterized by at least one radio interface, are deployed in the coverage area of the Macro-cell AP. Odin assumes that the controller can create VTPs as Virtual Machines (VMs) on a physical AP [5]. It is one kind of infrastructure-level virtualization. In this paper, VTPs are defined as virtual logical cells on the SDN controller. The cell virtualization is implemented using a dynamical interference graph partitioning method. In Fig. 1, two virtualized cells, VTP1 and VTP2, are created as logic entities on a controller based on graph partitioning. They share a common physical network but keep isolation as two independent virtualized cells.

In this scenario, we assume no application is running at the initial state of the system. When a mobile device is turned on, each of its radio interfaces scans the beacon frames heard from TPs and records the pair $<T P \_I d, r s s i \_v a l u e>$. The TP forwards the paired information as association_request through the Macro-cell AP to the SDN controller. A specific virtual association manager handles this association_request and then makes an association_decision based on the preferences of tenants. The manager will update the flow-table on the target_TP and notify the user with a specific target_TP_ID. The user will then send a Resource_request message to this specific TP and transmit data on the allocated resource. 


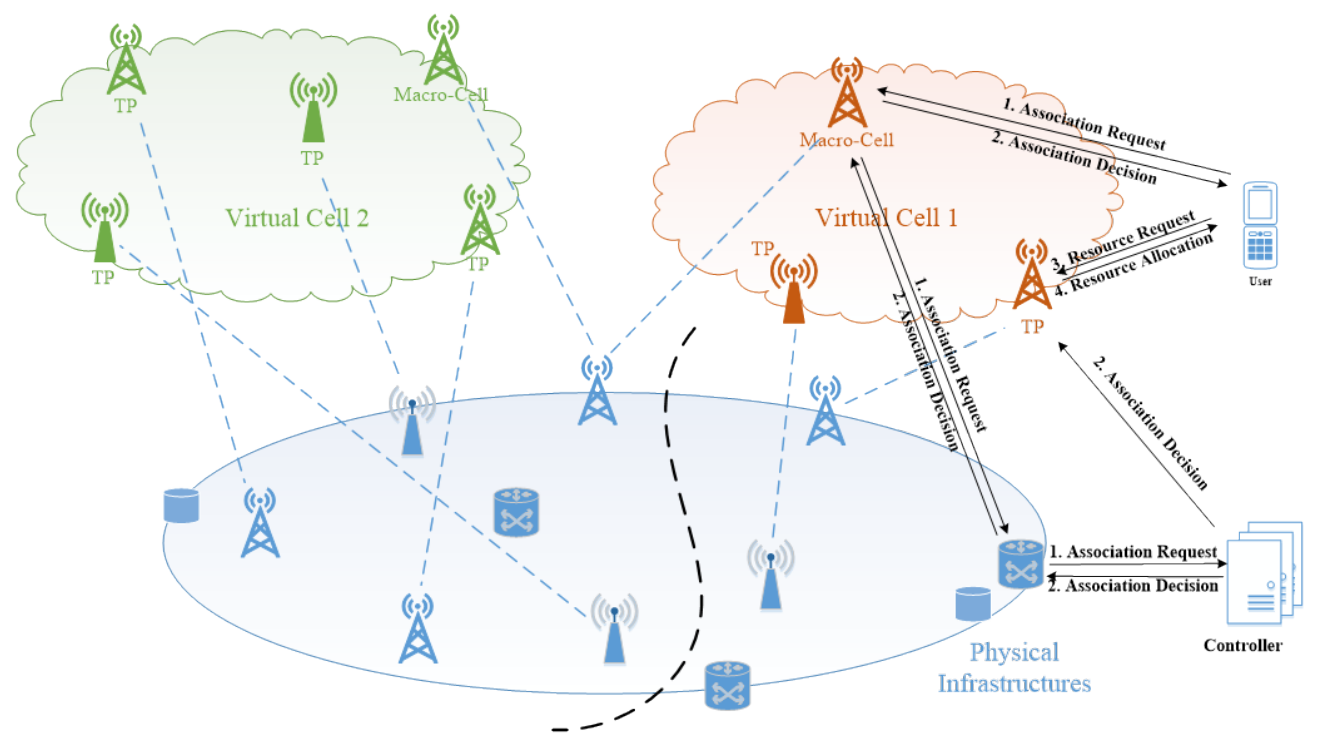

Fig. 1. The system architecture and signaling

Fig. 2 shows the software architecture in the SDN controller for association. The SDN controller has three modules, i.e. physical-TP association, cell virtualization and monitoring.

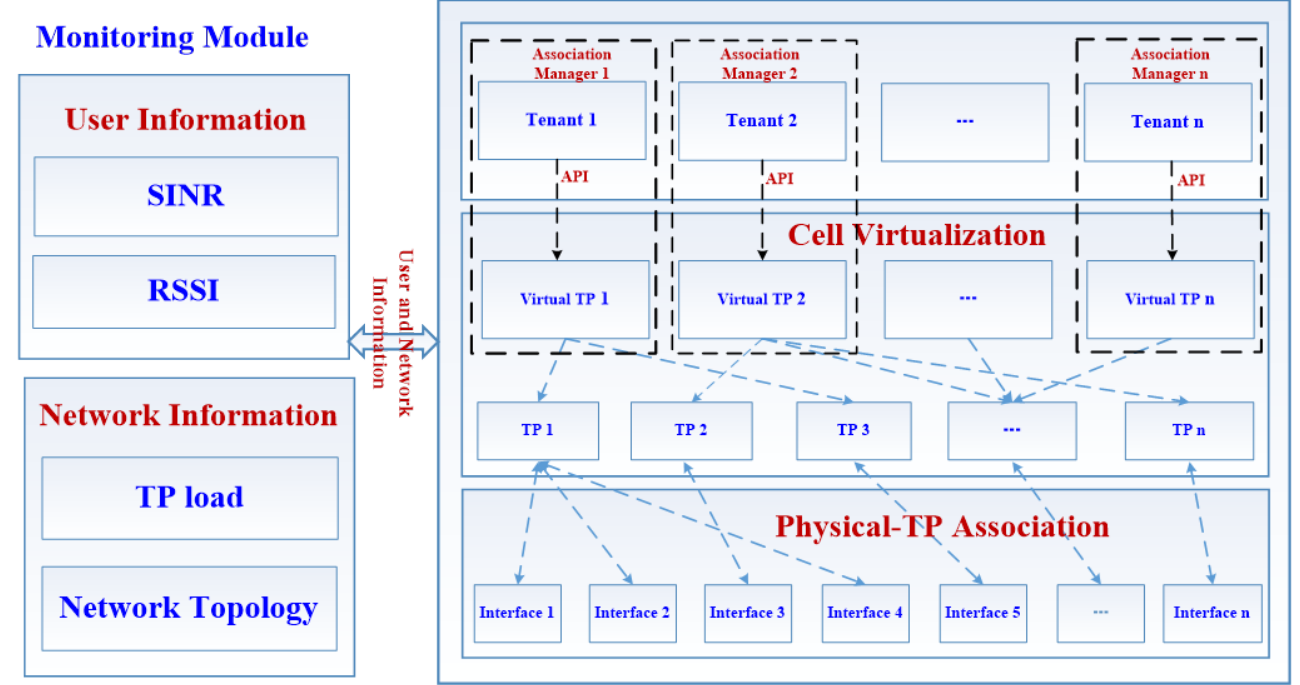

Fig. 2. The software architecture for association manager

In the monitoring module, user and network information are collected and then stored in the database. The user information includes a set of RSSI or SINR measurements. The network information includes load distribution on multiple TPs and network topology. In the cell virtualization module of the controller, multiple tenants co-exist and share all of the cells in the network. Each tenant will be allocated with one virtual cell. Therefore, each tenant gets a programmable network slice with a common API. The cell virtualization module dynamically matches the virtual cell to a set of physical TPs based on the graph partitioning algorithm. In each virtual cell, the module of physical-TP association dynamically matches each user to one or more specific TPs. Each tenant can define its own association manager based on its own preference.

The proposed framework provides programmable virtual cells, which can be customized for 
different tenants. Each tenant can define their own physical-TP association model based on its own preference. For example, in a high-definition video dispatching service, the objective of the user association is to maximize spectrum efficiency. For Voice over Internet Protocol (VOIP) service, the best association may be a coverage-oriented scheme. For a massive number of small packets in Internet of Things (IoTs), user association optimization may consider network latency. Therefore, cell virtualization based architecture enables a programmable association manager for different application scenarios for each tenant. In this paper, we provide physical cell association problem formulation with three examples in Section 3.4. We take the system or link capacity as an optimization objective where all TPs are fairly load balanced. Finally, the controller will make the decision on user association and notify users.

\subsection{Problem formulation}

We consider a densely deployed small cell network, composed of $J$ TPs and $U$ users, managed by an SDN controller. We assume that any user $u \in\{1,2, \ldots, U\}$ has $s_{u}$ radio interfaces. $I$ denotes the total number of all active radio interfaces in the network, $I=\sum_{u \in U} s_{u}$. The interference from other TPs to the radio communication link between interface $i$ and TP $j$ is $\sum_{k \neq j} P_{j} * l_{i k}$, where $P_{j}$ is the transmit power of TP $j$ and $l_{i k}$ is the channel gain from TP $k$ or TP $j$ to the interface $i$, which is interference. The SINR for the interface $i$ with its associated TP $j \in\{1,2, \ldots, J\}$ can be

$$
\gamma_{i j}=\frac{P_{j} * l_{i j}}{N_{0}+\sum_{k \neq j} P_{n} * l_{i k}}
$$

where $N_{0}$ is the power spectrum density of additive white Gaussian noise; $l_{i j}$ is the channel gain from TP $j$ to the interface $i$, which includes the path-loss and fading effects.

Let $a_{i j} \in\{0,1\}$ be the association indication variable between the interface $i$ and TP $j$, i.e $a_{i j}=1$,denotes the interface $i$ is associated with TP $j$; otherwise, the interface $i$ is not associated with TP $j$. Furthermore, we assume user demand on bandwidth is unlimited. Therefore, throughput of interface $i$ from $\mathrm{TP} j$ is:

$$
\Psi_{i j}=\frac{w_{j} * \log _{2}\left(1+\gamma_{i j}\right) * a_{i j}}{n_{j}}
$$

where $w_{j}$ is the bandwidth of TP $j$, and $n_{j}$ is the total number of interfaces associated with TP $j$. We formulate a Mixed Integer Nonlinear Programming (MINLP) problem to maximize the system throughput:

$$
\begin{gathered}
\max \sum_{j=1}^{J} \sum_{u=1}^{U} \sum_{i=1}^{S_{u}} \Psi_{i j} \\
\mathrm{C} 1: \sum_{j=1}^{J} a_{i j}=1, \forall i \in\{1,2, \ldots, I\} \\
\mathrm{C} 2: \sum_{i=1}^{S_{u}} a_{i j}=1, \quad \forall u \in\{1,2, \ldots, U\}, \forall j \in\{1,2, \ldots, J\}
\end{gathered}
$$

where C1 and C2 means that each user interface is connected to only one TP and all of the interfaces from the same user cannot be associated with the same TP respectively. The formulated problem is NP-hard and is not practical for a large scale network. Therefore, we resort to heuristic algorithms by dividing the formulated problem (2)-(5) into three steps: Interference graph construction virtual-cell association and physical-cell association. In order to create virtual-cell association in step 1, we need to group physical cells into a number of clusters, which are identified as virtual cells. In step 2, within a specific cluster of physical cells, we resort to optimal user association with load balancing. The virtual-cell association is 
preceded by an initial interference graph construction.

Considering radio coverage, a bipartite graph of network topology $G=\{\mathcal{J}, \mathcal{U}, E\}$ is a natural graph-based representation of the global network information, where $\mathcal{J}$ denotes the set of TPs and $\mathcal{U}$ denotes the set of users. Each edge $p_{u j}$ in the coverage graph is the average RSSI value measured by all interfaces of user $u$ for the signal coming from TP $j$. In general, the bipartite graph cannot be used for TP clustering, so we resort to a full-connectivity interference graph for TPs. Firstly, the Signal and Interference Ratio (SIR) $\gamma_{u, h \rightarrow g}$ for user $u$ in the coverage of TP $g$ with the interference from TP $h$ is as follows,

$$
\gamma_{u, h \rightarrow g}=\frac{P_{g} * l_{u g}}{N_{0}+P_{h} * l_{u h}}
$$

where $P_{g}$ and $P_{h}$ are the transmit power of TP $g$ and TP $h$, respectively. $l_{u g}$ is the channel gain between user $u$ and TP $g$ and $l_{u h}$ is the channel gain between user $u$ and TP $h$. We record the received signal strength for user $u$ from TP $g$ as $p_{u g}=P_{g} * l_{u g}$. Therefore, after ignoring thermal noise $N_{0}$, equation (6) is written as

$$
\gamma_{u, h \rightarrow g}=\frac{p_{u g}}{p_{u h}}
$$

where $p_{u g}$ denotes the RSSI for user $u$ and TP $g$, and $p_{u h}$ denotes the RSSI for user $u$ and TP $h$. Then, we can get the average SIR for all of users in the coverage of TP $g$ as

$$
C_{h \rightarrow g}=\sum_{u \in T P(g)} \frac{1}{|T P(g)|} \gamma_{u, h \rightarrow g}
$$

where $T P(g)$ is a set of users covered by TP $g$. $|T P(g)|$ is the number of users covered by TP $g$. Note that interference should be bidirectional. In the same way, we can get the formula of inter-cell SIR from TP $g$ to TP $h$ for any user $u$ in the coverage of TP $h$.

$$
\mathrm{C}_{g \rightarrow h}=\sum_{u \in T P(h)} \frac{1}{|T P(h)|} \gamma_{u, g \rightarrow h}
$$

where $\gamma_{u, h \rightarrow g}$ is equal to $\frac{p_{u h}}{p_{u g}}$ and $|\operatorname{TP}(h)|$ is the number of users in coverage by $\operatorname{TP} h$. TP(h) is a set of users covered by TP $h$.

Based on graph partitioning, we construct the undirected interference graph $\mathrm{G}=\{\mathcal{J}, E\}$ for TPs by combining two weights $C_{g \rightarrow h}$ and $C_{h \rightarrow g}$ of the bi-directional coverage graph as a common weight $e_{g h}$. Considering the relationship between two TPs by appraising inter-cell interference for all mobile users, $G$ is shown in Fig. 3. Finally, each edge in $G$ is defined as follows:

$$
e_{g h}=\sum_{u \in T P(g)} \frac{1}{|T P(g)|} \gamma_{u, h \rightarrow g}+\sum_{u \in T P(h)} \frac{1}{|T P(h)|} \gamma_{u, g \rightarrow h}, \forall g \neq h, \forall u
$$

where TP $g$ and TP $h$ are two arbitrary vertices in the graph G. $e_{g h}$ denotes the average SIR between TP $g$ and TP $h$.

We reformulate the cell virtualization problem into a graph partition problem in order to isolate the partitions of the network. We assume that $\mathcal{B}=\left\{B_{1}, B_{2}, \ldots, B_{M}\right\}$ is a partition of TP network $\mathcal{J}$, i.e. $B_{m_{1}} \cap B_{m_{2}}=\emptyset$ for $\forall m_{1} \neq m_{2}$ and $\cup_{m=1}^{M} B_{m}=\mathcal{J}$. $M$ is the total number of partitions of the TP network, which is known. We derive objective function (11) by maximizing the difference between the average SIR of intra-virtual-cell and inter-virtual-cell.

$$
\max \sum_{m_{1}=m_{2}} \sum_{g \in B_{m_{1}}, h \in B_{m_{2}}} e_{g h}-\sum_{m_{1}, m_{2}, m_{1} \neq m_{2}} \sum_{g \in B_{m_{1}}, h \in B_{m_{2}}} e_{g h}
$$

where $g$ and $h$ are two arbitrary vertices from $B_{j}$ and $B_{k} \cdot \sum_{m_{1}=m_{2}} \sum_{g \in B_{m_{1}}}, h \in B_{m_{2}} e_{g h}$ is the total weights of intra-virtual-cell edges and $\sum_{m_{1}, m_{2}, m_{1} \neq m_{2}} \sum_{g \in B_{m_{1}}, h \in B_{m_{2}}} e_{g h}$ is the total weights of inter-virtual-cell edges in the interference graph $G$. 


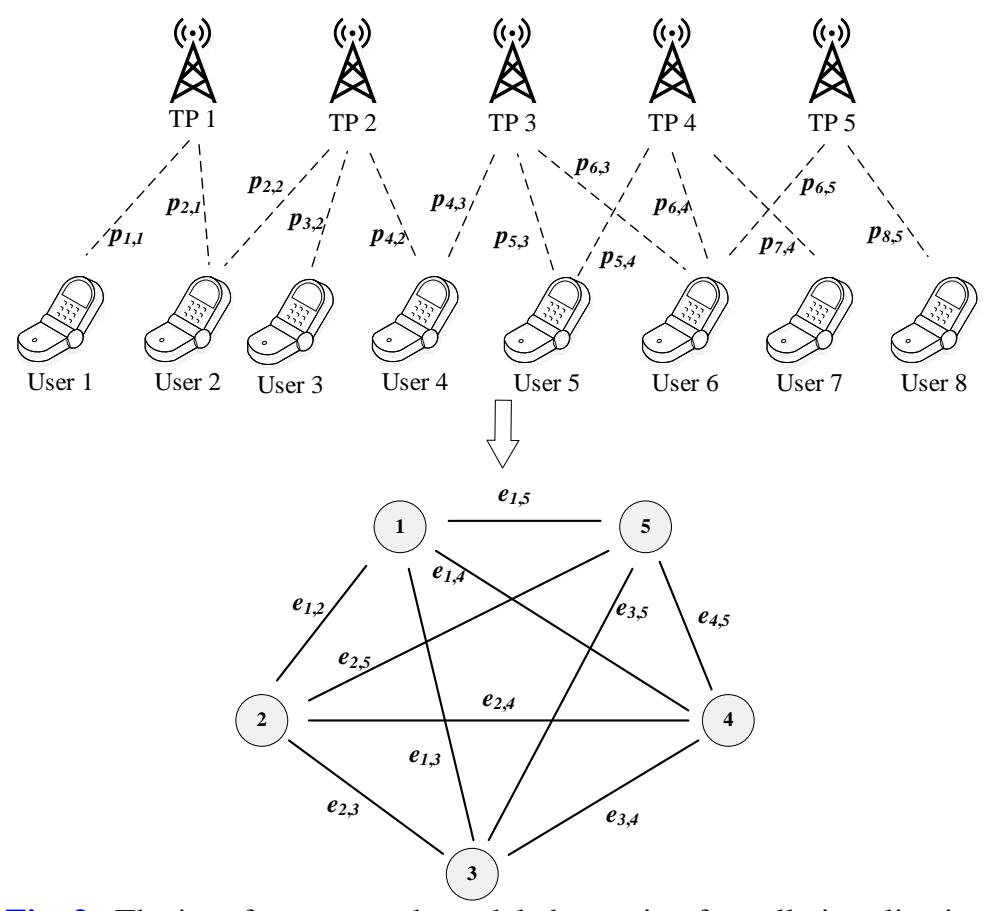

Fig. 3. The interference graph model abstraction for cell virtualization

\subsection{Virtual-cell association via Graph partition}

In this section, we propose an interference graph partitioning algorithm based on Kernighan-Lin Algorithm. By swapping two arbitrary vertices from two isolated partitions of the interference graph, we derive a utility function as the gain of swapping operation. We then minimize the inter-virtual-cell interference and the isolated independent virtual cell for each tenant. For each swapping operation, the corresponding utility function can be identified as:

$$
\begin{gathered}
\operatorname{gain}(g, h)=\left(\sum_{t \in G_{2}} e_{g t}-\sum_{t \in G_{1}} e_{g t}\right)+\left(\sum_{t \in G_{2}} e_{h t}-\sum_{t \in G_{1}} e_{h t}\right)-2 * e_{g h}, \\
g \in G_{1}, h \in G_{2}
\end{gathered}
$$

where $g$ and $h$ are two arbitrary vertices in $G_{1}$ and $G_{2}$, respectively. $\sum_{t \in G 1} e_{g t}$ is the internal cost of TP $g$ which is the sum of the costs of edges between TP $g$ and TP $t$ in $G_{1}$. $\sum_{t \in G 2} e_{g t}$ is the external cost of TP $g$ that is the sum of the costs of edges between TP $g$ and any TP $t$ in $G_{2}$.The cost is calculated based on the distance between the sum of intra-virtual-cell interferences inside a subgraph and the sum of inter-virtual-cell interference outside a subgraph. With this utility function, we find the maximum value of gain in any subgraph. The maximum value is achieved in the last iteration of the graph partitioning algorithm. We record the difference between internal cost and external cost as $Z_{g}=\left(\sum_{t \in G 1} e_{g t}-\sum_{t \in G 2} e_{g t}\right)$ for TP $g$ and $Z_{h}=\left(\sum_{t \in G 2} e_{h t}-\sum_{t \in G 1} e_{h t}\right)$ for TP $h$.

The whole procedure for the proposed algoritm is described in Fig. 4. The algorithm first computes the RSSI values between users and all TPs,which are taken as the raw data set. It then construct a weighted interference graph $G$, which takes each TP as the vertex and $e_{g h}$ in equation (10) as the edge between TP $g$ and TP $h$.The graph $G$ is partitioned into two equal sub-graphs $G_{1}$ and $G_{2}$ in order to maximize utility function in equation (12). The algorithm iterates and improves a partition using a greedy strategy to pair up vertices of $G_{1}$ and that of $G_{2}$ so that exchange of the paired vertices from one group to the other group will improve the 
sum of the partitioning gain defined in (12). Finally, it finds the maximum gain $g_{-}$max which is the sum of gain as shown in line 12 of Algorithm 1 in Fig. 4. In the proposed algorithm, the number of TPs is represented as $|V|$. In the KLA-based graph partitioning, the overall complexity of selecting a pair of TP is $O(|V| * \log (|V|))$. Since $|V| / 2$ exchanged pairs are selected in one loop of KLA, each iteration of the algorithm runs in time $O\left(|V|^{2} * \log (|V|)\right)$.

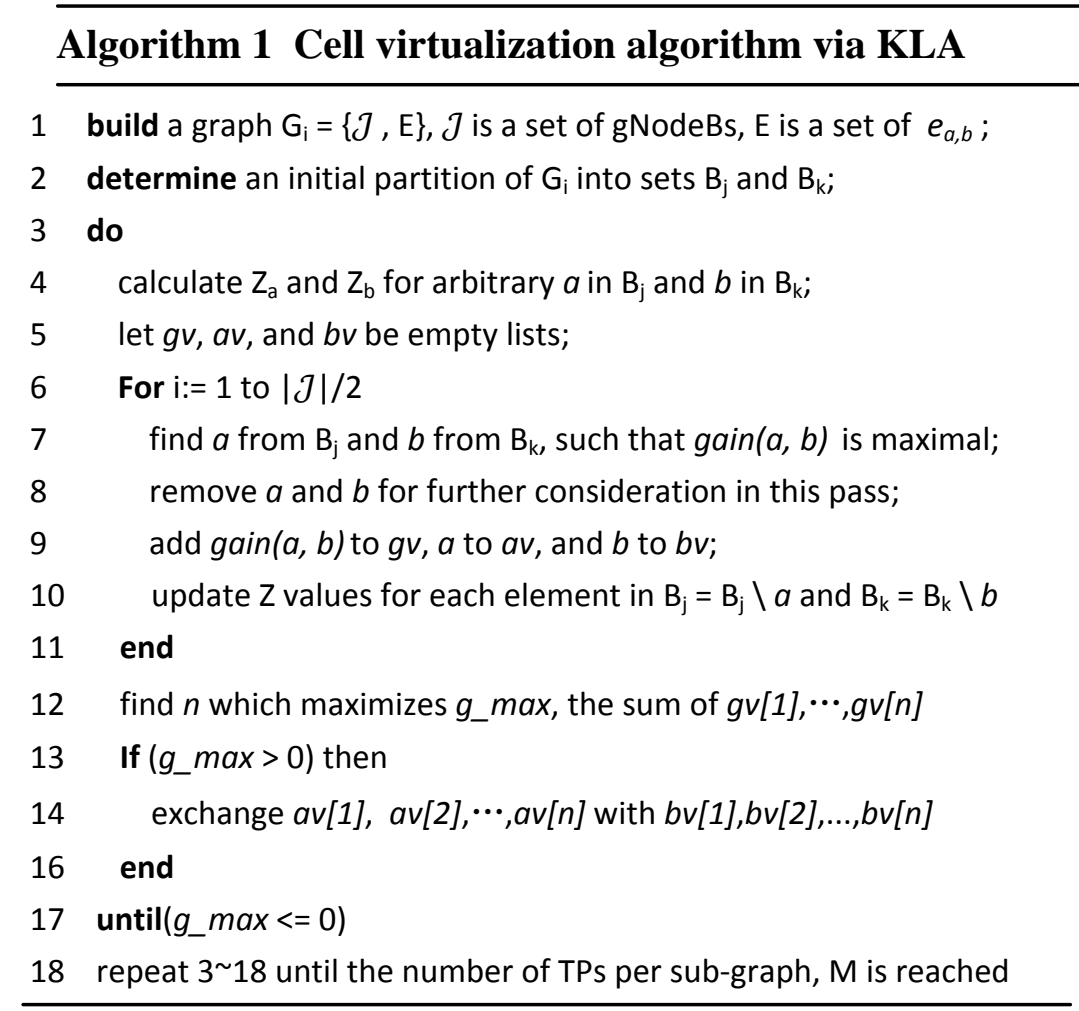

Fig. 4. The proposed association decision algorithm

\subsection{Customized Physical-cell association}

In the virtual-cell association, the association manager maps virtual cell $B_{m}$ with a group of physical TPs in a dynamic manner. However, in the physical-cell association, users will be associated to one or more specific TPs with the customized formulation. In this section, we consider the physical-cell association in one of virtual cells, $B_{m}$. In general, we assume each multi-homed user has $s_{u}$ radio interfaces. Here, let $i \in\{1,2, \ldots, I\}$ be the set of indexes of active interfaces in the system. User calculates $\overline{\mathbf{r}}_{i j}$ as the average RSSI value between the $i^{\text {th }}$ interface and the $j^{\text {th }} \mathrm{TP}$, where $i$ ranges from 1 to $I$ and $j$ ranges from 1 to $J$. Here, $\overline{\mathbf{r}}_{i j}$ is different with the $p_{i j}$, which is defined as the average RSSI value for user $i$ from TP $j$. To be simple, we estimate the link capacity $\Psi$ based RSSI measurements with a Shannon-like equation in [25]:

$$
\Psi=\delta * w * \log _{2}(\alpha+\beta * r s s i)
$$

where $\delta, \alpha$, and $\beta$ are coefficients to be determined by curve fitting based on the actual measurements in the realistic scenarios, and $w$ is the system bandwidth of TP. However, in a multi-user scenario for a TP, user throughput $\Psi_{i j}$ between the $j^{\text {th }}$ TP and the $i^{\text {th }}$ interface of user is 


$$
\begin{gathered}
\Psi_{i j}=\frac{w_{j} * \log _{2}\left(\alpha+\beta * \bar{r}_{i j}\right)}{n_{j}} \\
n_{j}=\sum_{i=1}^{I} a_{i j}, \forall j \in\{1,2, \ldots, J\}
\end{gathered}
$$

where, $n_{j}$ denotes the number of users being served by the $j^{\text {th }} \mathrm{TP}$, and $w_{j}$ denotes the system bandwidth of the $j^{\text {th }}$ TP. In order to show the feature of customized physical association in each virtual cell, we present and compare three formulations mapping the interfaces of users to the physical TPs dynamically.

(1) Formulation 1: Nearest-Neighbor association

For each virtual cell, Nearest-neighbor association manager will associate each interface with the nearest physical TP based on the RSSI measurements from users. The distance between the interface and physical TP is assumed to be an estimate of the RSSI measurements. The goal of the Nearest-Neighbor association is to maximize the individual link throughput based on RSSI measurements.

$$
\begin{gathered}
\Psi=\max \sum_{i=1}^{s_{u}} w_{j} * \log _{2}\left(\alpha+\beta * \bar{r}_{i j}\right) \\
\text { s.t. } \quad \sum_{i=1}^{s_{u}} a_{i j}=1, \forall u \in\{1,2, \ldots, U\}, \forall j \in\{1,2, \ldots, J\}
\end{gathered}
$$

where (17) means that all interfaces from the same user cannot be associated with the same TP. (2) Formulation 2: Pure maximum system throughput

Equation (18) defines the main goal of the proposed model which is to find the optimal association that maximizes the system throughput.

$$
\begin{array}{cc}
\Psi= & \max \sum_{i=1}^{I} \sum_{j=1}^{J} \frac{w_{j} * \log _{2}\left(\alpha+\beta * \bar{r}_{i j}\right)}{n_{j}} \\
\text { s.t. } & \sum_{j=1}^{J} a_{i j}=1, \quad \forall i \in\{1,2, \ldots, I\} \\
& \sum_{i=1}^{s_{u}} a_{i j}=1, \forall u \in\{1,2, \ldots, U\}, \forall j \in\{1,2, \ldots, J\}
\end{array}
$$

where constraint (19) denotes each user interface is connected to only one TP and constraint (20) denotes that interfaces from the same user cannot be associated to the same TP[25].

(3) Formulation 3: Optimal association with load balancing

Considering user fairness, load balancing among TPs is taken as a new constraint (24) for the objective function (21). The number of interfaces associated to each TP should be less than the average load.

$$
\Psi=\max \sum_{i=1}^{I} \sum_{j=1}^{J} \frac{w_{j} * \log _{2}\left(\alpha+\beta * \bar{r}_{i j}\right)}{n_{j}}
$$

s.t.

$$
\sum_{j=1}^{J} a_{i j}=1, \quad \forall i \in\{1,2, \ldots, I\}
$$

$$
\begin{gathered}
\sum_{i=1}^{s_{u}} a_{i j}=1, \forall u \in\{1,2, \ldots, U\}, \forall j \in\{1,2, \ldots, J\} \\
\sum_{i=1}^{I} a_{i j} \leq \frac{I}{J}, \forall j \in\{1,2, \ldots, J\}
\end{gathered}
$$

Because both formulation 2 and 3 are nonlinear integer programming problems, we solve it with a branch and boundary algorithm, provided by a MATLAB toolbox,YALMIP [26]. 


\section{Simulation Results}

\subsection{Scenario configuration}

In this section, the performance of the proposed algorithm with respect to the throughput is evaluated through simulations. All the experiments are performed in an indoor office scenario.

Fig. 5 (a) illustrates the scenario of physical-cell association. In this scenario, 5 TPs are deployed in a square area of $40,000 \mathrm{~m}^{2}$. The number of mobile users can increase from 5 to 60 . For a medium-scale network of 20 TPs, Fig. 5 (b) with dimensions $500 \mathrm{~m} \times 500 \mathrm{~m}$, we partition the network into 4 virtual cells for 4 tenants using KLA-based graph partition, as depicted in Algorithm 1. In each virtual cell, 5 independent cells are clustered dynamically and each user is assigned a maximum of two radio interfaces. The system bandwidth of each TP for data transmission is $20 \mathrm{MHz}$ with 5 TPs being deployed at the center of an area with a fixed radius about 200 meters. We also take an indoor office scenario into condieration. The relationship among RSSI, SINR and link capacity is modeled with measurements in an office environment.The model is built based on the curve fitting, as described in Section 3.4. We calculate the coefficient $\alpha, \beta$ and $\delta$ in Equation (13) with this curve fitting. RSSI measurements are derived from the path-loss model, which is defined as:

$$
\text { PathLoss = 20lg(E)-20lg(D)-32.4 }
$$

where the carrier frequency $E$ is set at $2.4 \mathrm{GHz}$, and $D$ is the distance between the mobile user and TPs.The PathLoss is used to calculate RSSI value as follows:

$$
\text { RSSI }=\text { P- } S \text { - PathLoss }
$$

where $P$ is the transmit power of TP, which is set as $-10 \mathrm{dBm}$, and $S$ is the shadowing effect of obstacles in the network. $S$ is choosen randomely betwwen 0 and 40 in $\mathrm{dB}$.

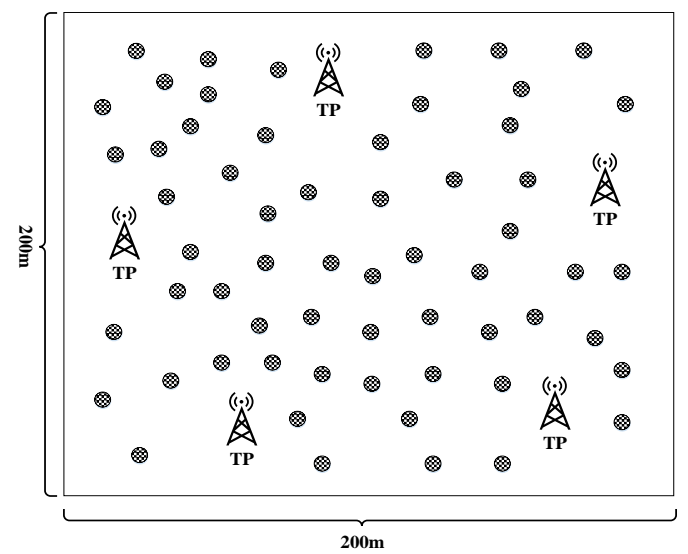

(a)

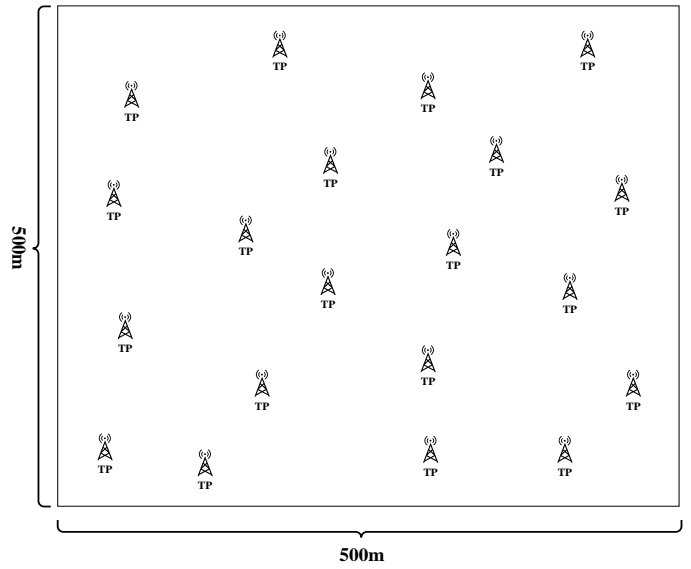

(b)

Fig. 5. Scenarios of small-cell deployment

\subsection{Model-oriented parameter fitting}

In order to determine the parameter $\alpha, \beta$ and $\delta$ in Equation (13), we establish the relationship between RSSI and achieved throughput with actual measurements in an office environment, which is the curve of "realistic measurements" in Fig. 6. By the model-based curve fitting in MATLAB, we get $\alpha=1.733, \beta=0.005429$, and $\delta=5.0$. The comparison illustrated in Fig. 6 
shows that the model-based curve fitting matches the realistic measurements well. Therefore, Equation (13) can be adopted as the model of user throughput.

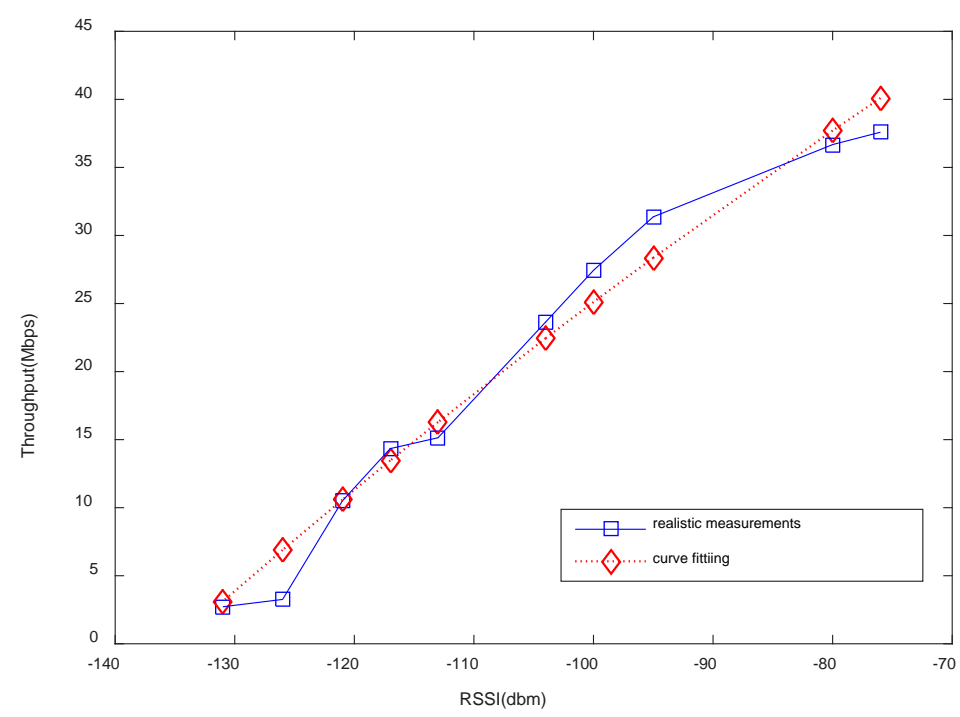

Fig. 6. Model-based curve fitting with realistic measurements

The proposed physical-cell association control is evaluated in a defined scenario, as shown in Fig. 5(a). Three association schemes are compared: Nearest-Neighbor association, Pure Maximum Throughput scheme and Load Banlancing scheme. In the performance evaluation of the physical-cell association, 3 metrics are considered: system throughput, user experience and user fairness. A common configuration list is summarized in Tab. 1.

Tab. 1: PARAMETERS CONFIGURATION

\begin{tabular}{|l|l|}
\hline Parameter and Units & Setting Values \\
\hline Number of TPs & 5 or 20 \\
\hline Number of users & dynamic, 5 60 \\
\hline Number of interface & 1 or 2 \\
\hline Radius of coverage(m) & 200 \\
\hline Distance of neighbor TPs(m) & random \\
\hline Frequency band(GHz) & 2.4 \\
\hline Transmit power(dBm) & -10 \\
\hline Shadowing effects(dB) & $(0,40)$ \\
\hline Bandwidth $(\mathrm{MHz})$ & 20 \\
\hline The coefficient $\alpha$ & 1.733 \\
\hline The coefficient $\beta$ & 0.005429 \\
\hline The coefficient $\delta$ & 5 \\
\hline RSSI threshold for Edge user $(\mathrm{dBm})$ & -120 \\
\hline
\end{tabular}

\subsection{Virtual-cell association: Static scenario}

To solve the virtual-cell association problem, we choose the graph partition algorithm (dynamic KLA-based network partitioning, which is proposed in this paper) due to the computational complexity of MINLP problem. The number of clusters $K$ is 4 . Here, the 
performance of the proposed algorithm is compared with other schemes i.e a fixed clustering in a Cloud-RAN[8] and the dynamic user clustering for minimum Intra-Cluster Interference (ICI)[27]. Fig. 7 shows results for virtual-cell association for three different algorithms. 'o' represent TPs, and '*' represent users. The result in (a) is achieved by fixed clustering in Cloud-RAN, the result in (b) is achieved by the KLA dynamic partitioning. Different colors are used for TPs and users in different VTP set. The result in (c) is achieved by minimum ICI clustering.

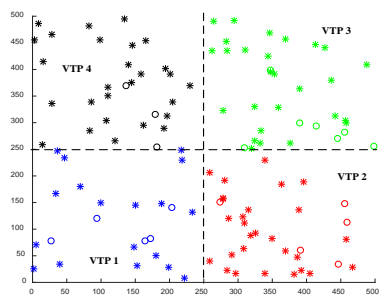

(a) Fixed

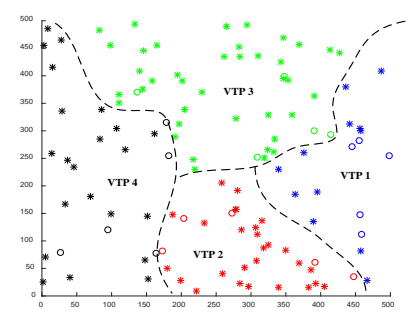

(b) KLA

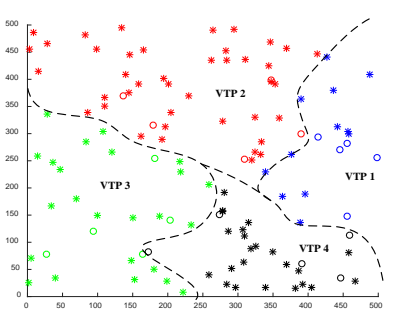

(c) ICI

Fig. 7. The virtual-cell association results

The effect on throughput of each VTP for three schemes is shown in Fig. 8. The KLA based graph partitioning algorithm achieves load balancing for each independent VTP, as it can adapt to dynamic user distribution with an equal number of physical TPs. However, the virtual-cell association in Cloud-RAN with fixed partitions leads to the degradation of throughput for some VTPs. That is because that it is a static clustering scheme while losing the correlation information of TPs and user distribution. The Minimum ICI scheme is still not better than KLA. It considers intra-cluster interference only while KLA considers the utility gain function. Therefore, the KLA algorithm can achieve mapping from virtual TP to physical TPs with the interference graph. It can achieve a load-balanced traffic distribution among physical TPs in a large scale network.

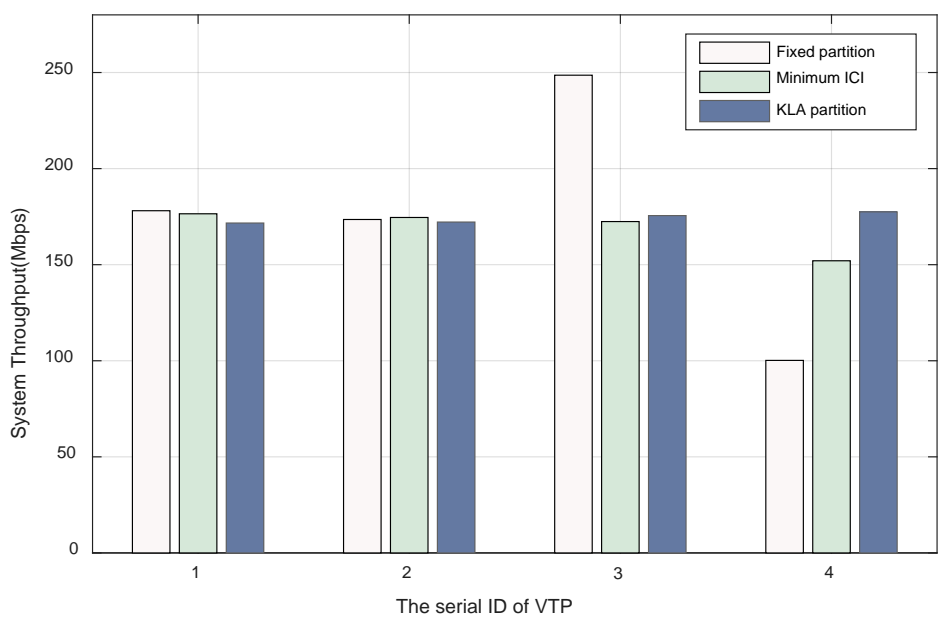

Fig. 8. Virtual-cell association algorithm comparison on system throughput

In order to evaluate the scalability of system throughput for the proposed KLA-based algorithm, we increase the number of TPs in the area from 8 to 40, and maintain the total number of users at 100. The results of the three algorithms are shown in Fig. 9. The system 
throughput benefits from the dense-deployment of small cells, although it results in inter-cell interference. $K$ represents the number of TPs in one virtual cell. In this simulation, we set $K$ as 4 and 8. With the number of TP increases, the proposed KLA algorithm outperforms the minimum ICI scheme. Although the fixed partition still has a high system throughput, it results in unbalanced load distribution as shown in Fig. 8. In addition, the system throughput becomes higher with the increased number of TPs, as shown in Fig. 9.

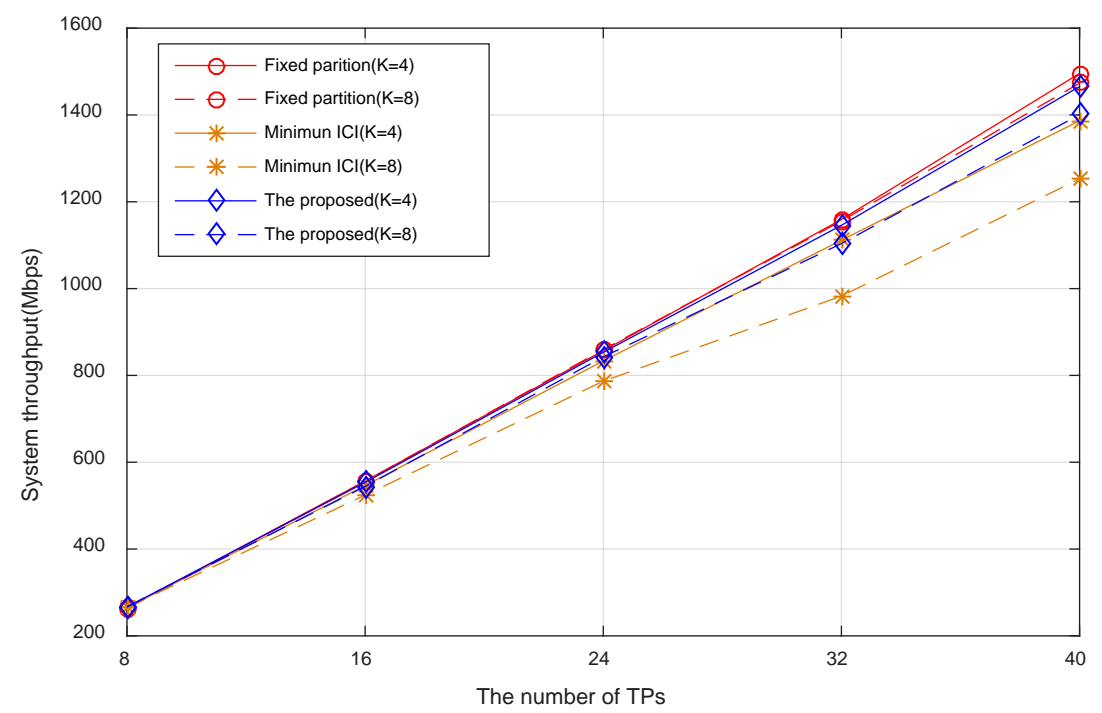

Fig. 9. The algorithm comparison with various network scales

\subsection{Virtual-cell association: Mobility scenario}

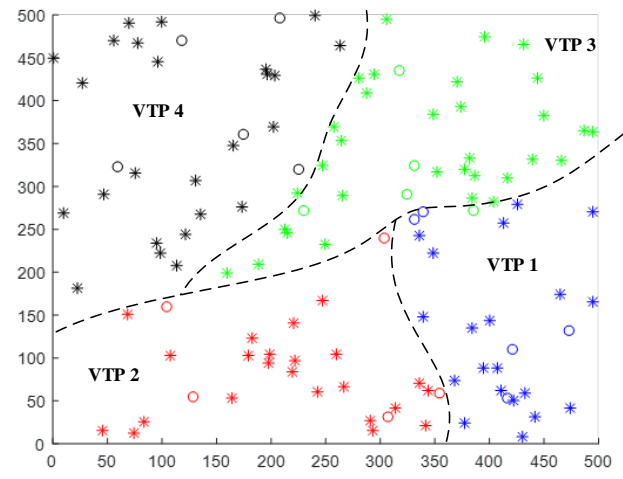

(a)

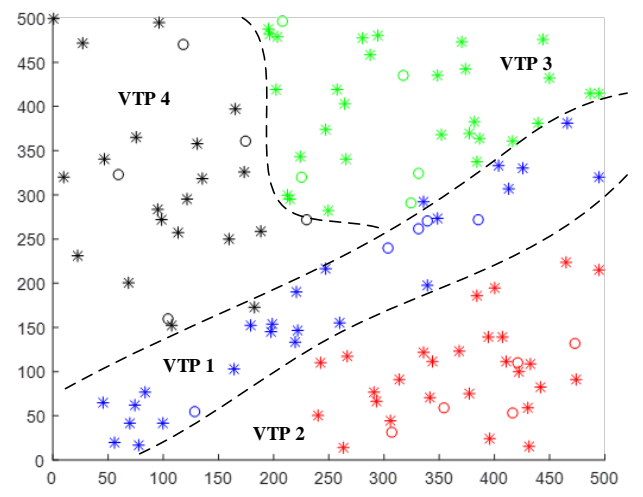

(b)

Fig. 10. The KLA-based results in mobility scenarios

Based on the results shown in Section 4.3, it is established that KLA-based graph partition works in a self-organized manner. In this section, we consider mobility scenario in Fig. 10. The user location in Fig. 10(a) is generated uniformly in the area of 500x500 meters. In Fig. 10(b), all users in Fig. 10(a) are moved 50 meters above the Y-axis. Those users which lies outside 50 meters will have to re-enter from the base of the Y-axis. As shown in Fig. 11, we check the system throughput and find the average user throughput in this dynamic scenario with user mobility. Although the mapping between VTP and physical TPs are changed 
because of user mobility, system throughput and average user throughput for each VTP does not change. The user experience is much better than their bandwidth demands. More detailed simulation resultsfor each user will be examined in the physical-cell association in the following sections.

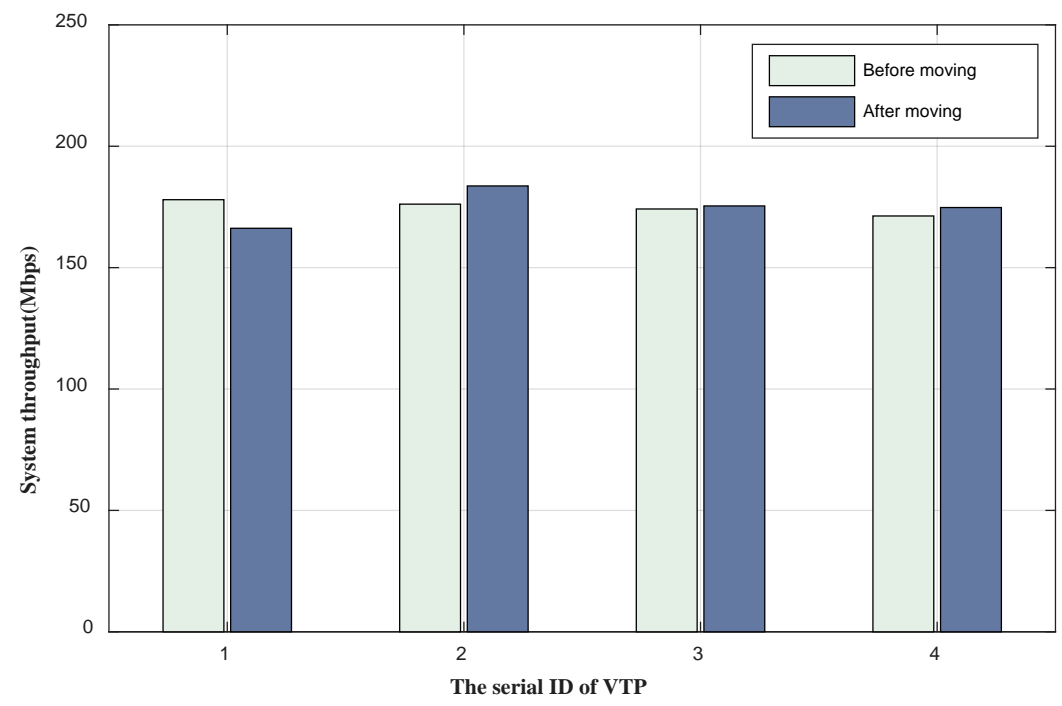

(a) System throughput

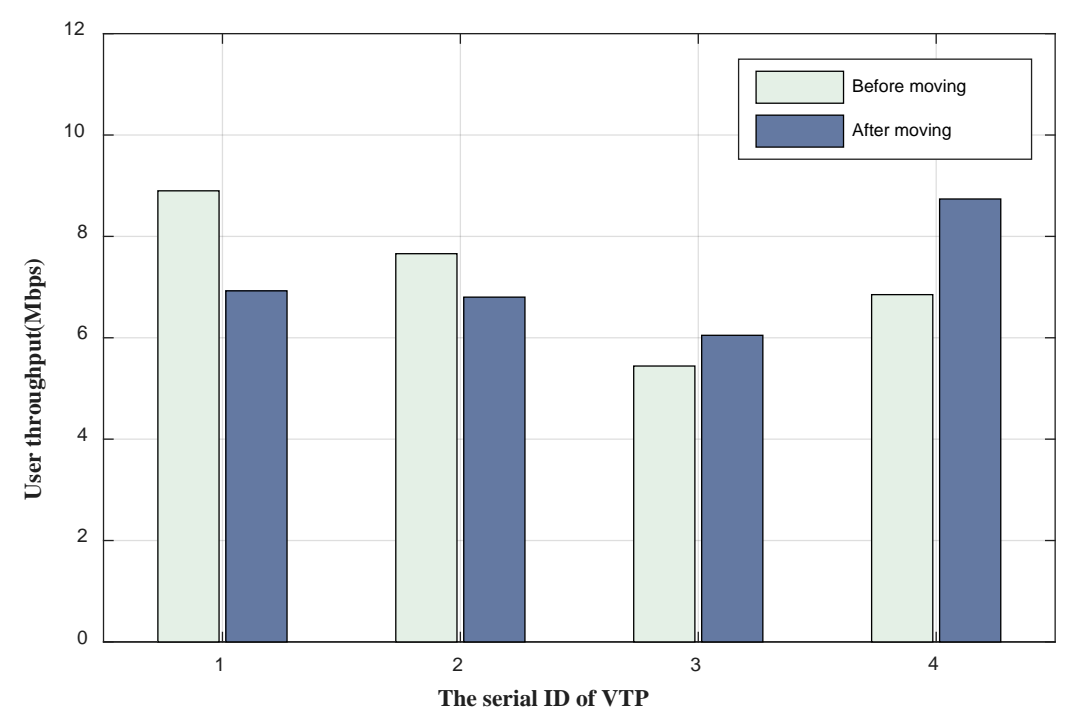

(b) User throughput

Fig. 11. Throughput comparison on mobility scenario

\subsection{Physical-cell association: System throughput}

We assume each user has a single radio interface, $s_{u}=1$ in the constraint of Equation (17), (20) and (23). It is reasonable to assume each mobile user has one flow, and each flow has an unlimited bandwidth requirement. The performance of system throughput is considered for three association approaches. The initial user distribution in the area is uniform. The receiver 
sensitivity of each user is configured with $-120 \mathrm{dBm}$. The performance comparison is illustrated in Fig. 12.

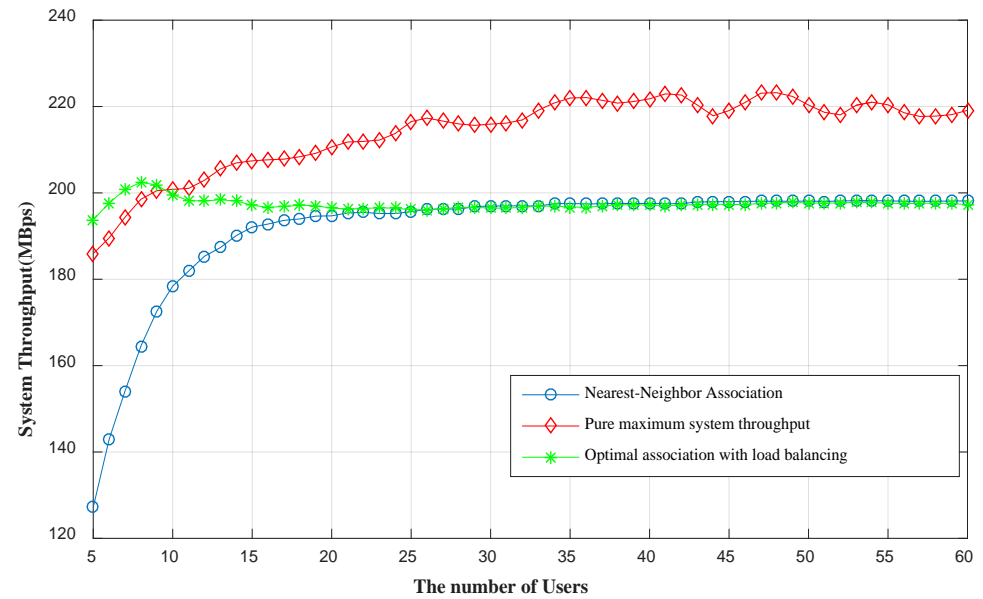

Fig. 12. The performance on system throughput

With lots of snapshots in the simulation, Pure Maximum Throughput association can achieve the best system throughput by satisfying the user in a good quality channel. However, load distribution among multiple TPs is not balanced. Therefore, we consider load balancing as an additional constraint for the optimal association in formulation 3. As illustrated in Fig. 12, Pure Maximum Throughput algorithm has the best system throughput, when the number of users are more than 10 . The optimal association with load balancing achieves a higher system throughput for 10 users, but has identical systems throughput with Nearest Neighbor RSSI-based association for more than 20 users.

\subsection{Physical-cell association: User experience}

In Fig. 13, the individual throughput goes down when the number of users increases from 5 to 60 in the scenario of Fig. 5(a). Especially, formulation 3 performs better on individual throughput than the other two association schemes. However, Pure Maximum Throughput association is not reliable with heavy fluctuations. Therefore, the Pure Maximum Throughput association leads to the worst user experience in terms of individual throughput.

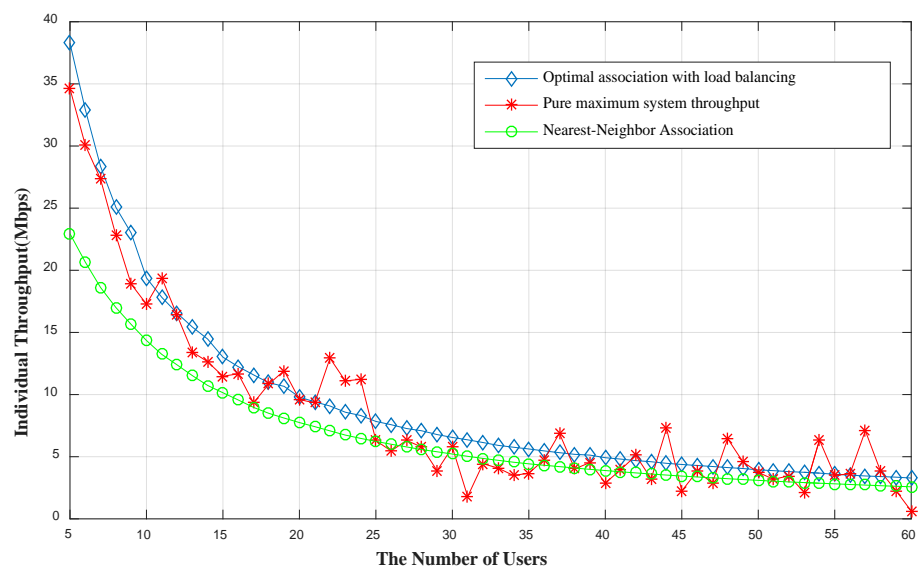

Fig. 13. The performance on individual throughput 


\subsection{Physical-cell association: User fairness}

In addition to throughput, we still need to consider fairness among different users. The fairness index is defined as the standard variance of individual throughput. A high fairness index indicates unfairness among users. As shown in Fig. 14, the optimal association with load balancing approach performs better. Pure Maximum Throughput association, denoted in red curve, has the worst fairness.

$$
\text { Fairness Index }=\sqrt[2]{\left(\Psi_{\max }-\frac{\Psi_{\max }+\Psi_{\min }}{2}\right)^{2}+\left(\Psi_{\min }-\frac{\Psi_{\max }+\Psi_{\min }}{2}\right)^{2}}
$$

where $\Psi_{\max }$ is the maximum user throughput and $\Psi_{\min }$ is the minimum user throughput.

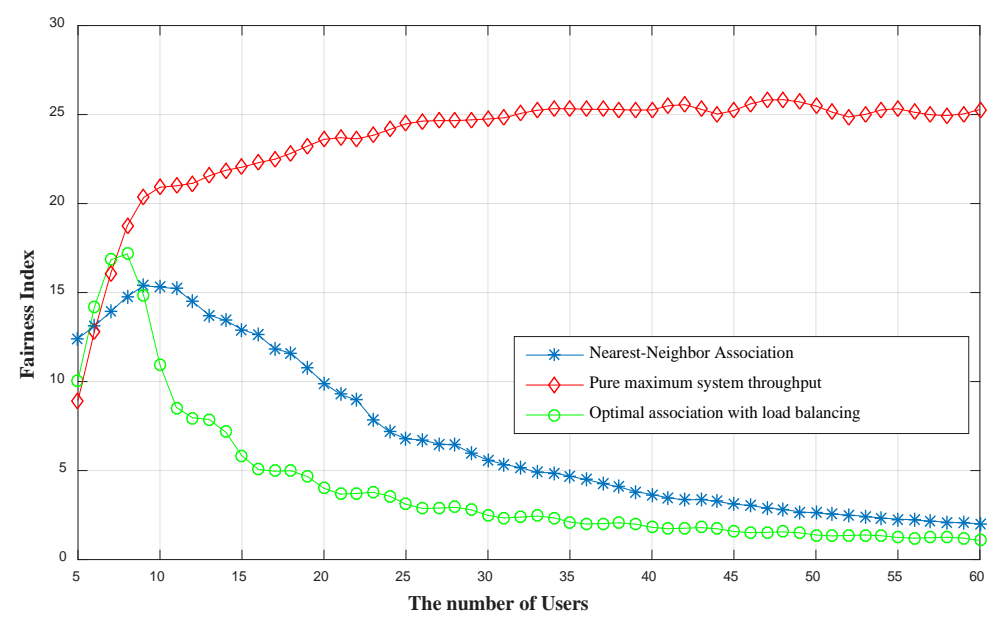

Fig. 14. The performance on user fairness

\subsection{Physical-cell association: Impact of multi-homing}

Previously, mobile users are assumed to have one radio interface. In this section, we analyze the individual throughput in a scenario, in which mobile users can be equipped with either single-interface or double-interface. In this simulation, the total number of users is 30 and the number of double-interface users changes from 0 to 30. The results are illustrated in Fig. 15 and Fig. 16. In Fig. 15, the average individual throughput decreases a little with the number of double-interface users increases. Meanwhile, double-interface users always achieve higher individual throughput than single-interface users. 


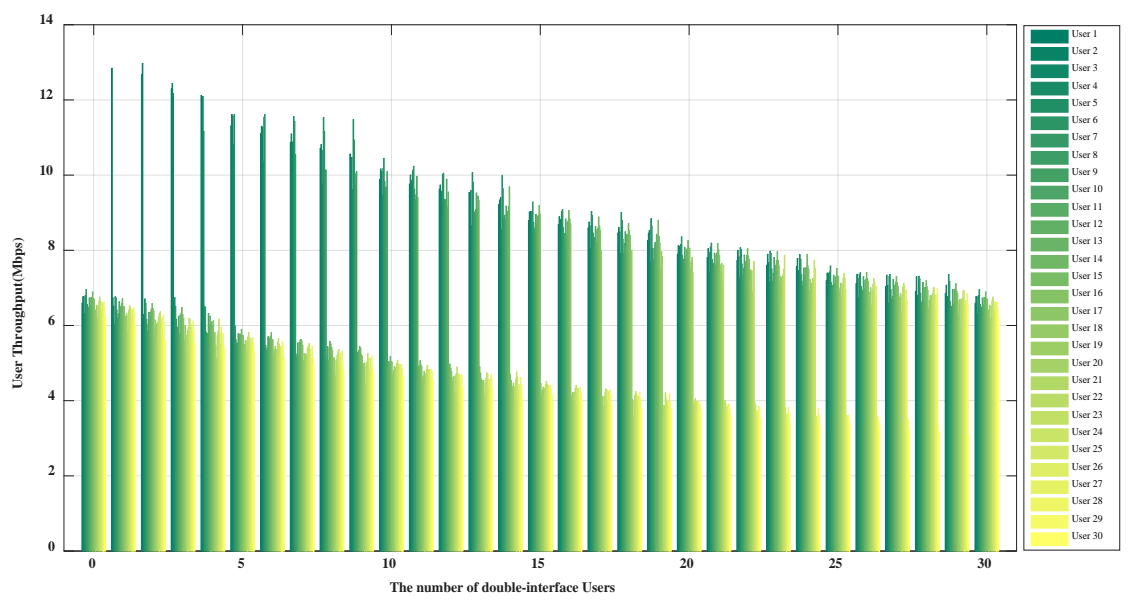

Fig. 15. Individual throughput with a mixed number of interfaces

Furthermore, we recorded the minimum value of individual throughputs for double-interface users in "white" pillar, and the maximum value of individual throughputs for single-interface users in "black" pillar. The results, as illustrated in Fig. 16, verify that the double-interface users can achieve a larger throughput than the single-interface users.

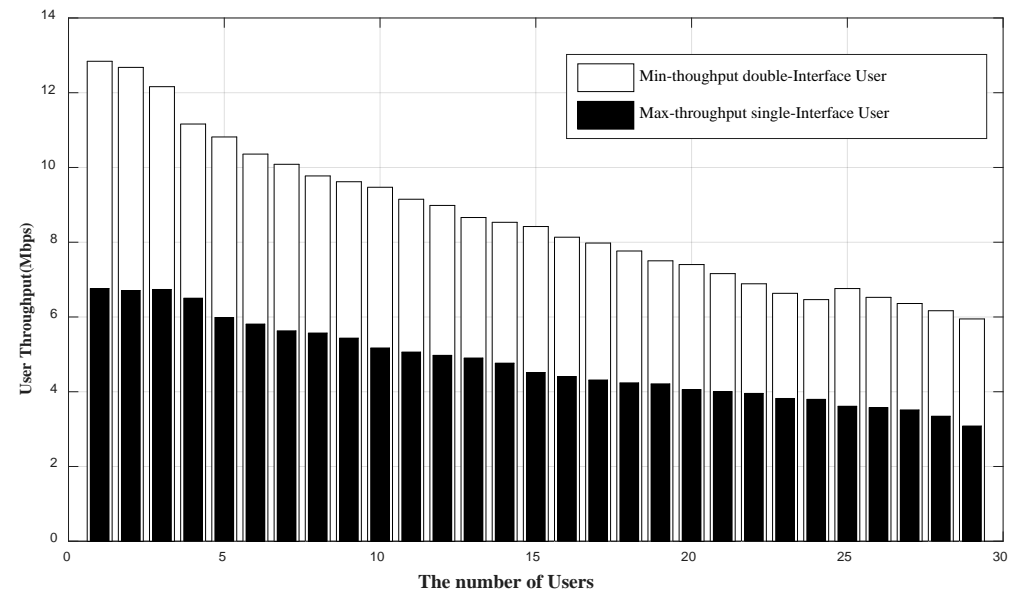

Fig. 16. The comparison of individual throughput

(Double-interface vs. Single-interface)

\section{Conclusion}

In this paper, a framework of cell virtualization and customized user association has been proposed in a multiple-tenant scenario. An interference graph partitioning algorithm is proposed for cell virtualization in software defined small cell networks. The simulation results justified that the proposed virtual-cell association can achieve the better performance in terms of throughput. For multi-homed users, three formulations of physical-cell association are customized and compared. The load balancing association outperforms other schemes in term of system throughput and user fairness. 


\section{Acknowledgment}

This work is supported by the National Nature Science Foundation of China under Grant no. 61771098, by the Science and Technology Planning project of Sichuan Province, China, under grant no. 2016GZ0075, by the Fundamental Research Funds for the Central Universities under grant no. ZYGX2014J060, and the ZTE Innovation Research Fund for Universities Program 2016.

\section{References}

[1] Vu Q D, Tran L N, Juntti M, et al. "Energy-efficient bandwidth and power allocation for multi-homing networks,” IEEE Transactions on Signal Processing, vol. 63, no. 7, pp. 1684-1699, 2015. Article (CrossRef Link)

[2] Sun G, Eng K, Yin S, et al. "MARS: multiple access radio scheduling for a multi-homed mobile device in Soft-RAN,” KSII Transactions on Internet \& Information Systems, vol. 10, no.1, pp. 79-95, 2016. Article (CrossRef Link)

[3] D. Liu, L. Wang, Y. Chen, M. Elkashlan, et al., "User association in 5G networks: a survey and an outlook,” IEEE Communications Surveys \&Tutorials, vol.18, no.2 pp.1018-1044, 2016. Article (CrossRef Link)

[4] M. Mendonc, B. N. Astuto, X. N. Nguyen, K. Obraczka, and T. Turletti., "A survey of software-defined networking: past, present and future of programmable networks," IEEE Communications Surveys \& Tutorials, vol. 16, no. 3, pp. 1-18 , 2014. Article(CrossRef Link)

[5] S. Lalith, S.-Z. Julius, M. Ruben, F. Anja, and V. Teressa, "Towards programmable enterprise WLANS with Odin," in Proc. of Proceedings of the first workshop on Hot topics in software defined networks (HotSDN), 2012, pp.115-120. Article(CrossRef Link)

[6] P. Dely, J. Vestin, A. Kassler, N. Bayer, H. Einsiedler, C. Peylo, “Cloud-MAC- an OpenFlow based architecture for 802.11 MAC layer processing in the cloud,” in Proc. of IEEE Globecom Workshops, pp.186-191, 2012. Article(CrossRef Link)

[7] Blenk, A., et al. "Survey on network virtualization hypervisors for software defined networking," IEEE Communications Surveys \& Tutorials, vol. 18, no. 1, pp. 655-685, 2016.

Article(CrossRef Link)

[8] H. zhang, W. Wang, X.Li and H.Ji, "User association scheme in Cloud-RAN based small cell network with wireless virtualization," in Proc. of IEEE INFOCOM, pp. 384-389, 2015. Article(CrossRef Link)

[9] Chunguo Li, Peng Liu, Chao Zou, Fan Sun, John M. Cioffi, and Luxi Yang, "Spectral-efficient cellular communications with coexistent one- and two-hop transmissions," IEEE Transactions on Vehicular Technology, vol. 65, no. 8, pp. 6765-6772, Aug. 2016. Article(CrossRef Link)

[10] Chunguo Li, Hyun Jong Yang, Fan Sun, John M. Cioffi, and Luxi Yang, "Adaptive overhearing in two-way multi-antenna relay channels,” IEEE Signal Processing Letters, vol. 23, no. 1, pp. 117-120, Jan. 2016. Article(CrossRef Link)

[11] Kim W. "Dual connectivity in heterogeneous small cell networks with mmWave backhauls," Mobile Information Systems, vol.2016, no. 7, pp. 1-14, 2016. Article(CrossRef Link)

[12] J. Andrews, S. Singh, Q. Ye, X. Lin, and H. Dhillon, “An overview of load balancing in HetNets: old myths and open problems,” IEEE Wireless Communications, vol. 21, no. 2, pp. 18-25, April 2014. Article(CrossRef Link)

[13] Chunguo Li, Kang Song Dongming Wang, Fu-Chun Zheng, Luxi Yang, "Optimal remote radio head selection for cloud radio access networks,” Science China Information Sciences, vol. 59, no. 10, Oct. 2016. Article(CrossRef Link)

[14] H.-S. Jo, Y. J. Sang, P. Xia, and J. Andrews, "Heterogeneous cellular networks with flexible cell association: A comprehensive downlink sinr analysis," IEEE Transactions on Wireless Communications, vol. 11, no. 10, pp. 3484-3495, Oct. 2012. Article(CrossRef Link) 
[15] S. Corroy, L. Falconetti, and R. Mathar, "Dynamic cell association for downlink sum rate maximization in multi-cell heterogeneous networks," in Proc. of Proceedings of IEEE International Conference on Communications (ICC'12), pp. 2457-2461, Jun. 2012. Article(CrossRef Link)

[16] H. Kim, G. de Veciana, X. Yang, and M. Venkatachalam, "Distributed alpha-optimal user association and cell load balancing in wireless networks," IEEE/ACM Transactions on Networking, vol. 20, no. 1, pp. 177-190, Feb. 2012. Article(CrossRef Link)

[17] T. Han and N. Ansari, "User association in backhaul constrained small cell networks," in Proc. of 2015 IEEE Wireless Communications and Networking Conference(WCNC), pp.1637-1642, 2015. Article (CrossRef Link)

[18] T. Han and N. Ansari, "Green-energy aware and latency aware user associations in heterogeneous cellular networks," in Proc. of Proceedings of IEEE Global Telecommunications Conference (GLOBECOM'13), Atlanta, GA, USA, Dec. 2013. Article(CrossRef Link)

[19] A. Mesodiakaki, F. Adelantado, L. Alonso, and C. Verikoukis, "Energy efficient context-aware user association for outdoor small cell heterogeneous networks," in Proc. of IEEE Int. Conf. Commun. (ICC), pp. 1614-1619, Jun. 2014. Article(CrossRef Link)

[20] Chunguo Li, Yanshan Li, Kang Song, "Energy efficient design for multiuser downlink energy and uplink information transfer in 5G," Science China Information Sciences, vol. 59, no. 2, Feb. 2016. Article(CrossRef Link)

[21] H. Dhillon, R. Ganti, F. Baccelli, and J. Andrews, "Modeling and analysis of K-tier downlink heterogeneous cellular networks,” IEEE J. Sel. Areas Commun., vol. 30, no. 3, pp. 550-560, Apr. 2012. Article(CrossRef Link)

[22] H. Dhillon, R. Ganti, and J. Andrews, "Load-aware modeling and analysis of heterogeneous cellular networks,” IEEE Trans. Wireless Commun., vol. 12, no. 4, pp. 1666-1677, Apr. 2013. Article (CrossRef Link)

[23] K.Zarifi, H.Baligh, J.Ma, M.Salem and A.Maaref, "Radio access virtualization: cell follows user," IEEE PIMRC, pp. 1381-1385, 2014. Article(CrossRef Link)

[24] Y. Ruyue Li, P. Hao, F. Xie, H. Xiao and M. Ren, "Cell and user virtualization for ultra-dense network,” IEEE PIMRC, pp. 2359 - 2363, 2015. Article(CrossRef Link)

[25] J. Shang, L. Yu, C. Xue and W. Li, "Optimizing AP association in wireless mesh network with multipath TCP," in Proc. of Proceedings of the 21st IEEE International Workshop on Local and Metropolitan Area Networks (IWLANMAN), pp.1 - 6, 2015. Article(CrossRef Link)

[26] Lofberg, J.,"YALMIP: a toolbox for modeling and optimization in MATLAB," in Proc. of IEEE International Symposium on Computer Aided Control Systems Design, pp. 284-289, 2004. Article(CrossRef Link)

[27] Pateromichelakis E, Shariat M, Quddus A, et al, "Dynamic clustering framework for multi-cell scheduling in dense small cell networks," IEEE Communications Letters, vol. 17, no. 9, pp. 1802-1805, 2013. Article(CrossRef Link)

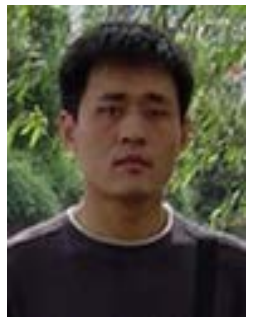

Guolin Sun received his B.S., M.S. and Ph.D. degrees all in Comm. and Info. System from the University of Electronic Sci.\&Tech. of China (UESTC), Chengdu, China, in 2000, 2003 and 2005 respectively. After Ph.D. graduation in 2005, Dr. Guolin has got eight years industrial work experiences on wireless research and development for LTE, Wi-Fi, Internet of Things (ZIGBEE and RFID, etc.), Cognitive radio, Location and navigation. Before he join the School of Computer Science and Engineering, University of Electronic Sci.\&Tech. of China, as an Associate Professor on Aug. 2012, he worked in Huawei Technologies Sweden. Dr. Guolin Sun has filed over 30 patents, and published over 30 scientific conference and journal papers, acts as TPC member of conferences. Currently, he serves as a vice-chair of the 5G oriented cognitive radio SIG of the IEEE (Technical Committee on Cognitive Networks (TCCN) of the IEEE Communication Society. His general research interest is 5G/2020 oriented wireless network, such as software defined networks, network function virtualization, wireless networks. 

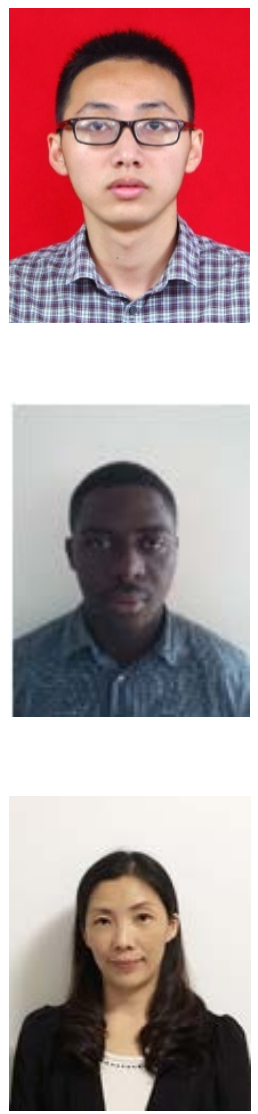

Xiufen Fang received his B.S. degree and M.S. degree in Department of Applied Mathematics both from the University of Electronic Science and Technology of China, Chengdu, China, in 1995 and 2001, respectively. She was a visiting scholar at Northern Illinois University (NIU) during March to September in 2012. Now, she is an associate professor in the School of Mathematical Sciences, University of Electronic Science and Technology of China. Her research interests include big data, neural networks and scientific computing.

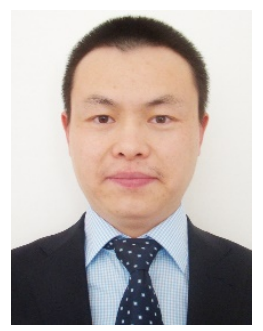

Wei Jiang received his Ph.D degree from Beijing University of Posts and Telecommunications (BUPT) in 2008. Since Mar. 2008, he has been worked 4 years in Central Research Institute of Huawei Technologies, in the field of wireless communications and 3GPP standardization. In Sept. 2012, he joined the Institute of Digital Signal Processing, University of Duisburg-Essen, Germany, where he was a Postdoctoral researcher and worked for EU FP7 ABSOLUTE project and H2020 5G-PPP COHERENT project. Since Oct. 2015, he joined the Intelligent Networking Group, German Research Center for Artificial Intelligence (DFKI), Kaiserslautern, Germany, as a senior researcher and works for H2020 5G-PPP SELFNET project. Meanwhile, he also works for the Department of Electrical and Information Technology (EIT), Technische University (TU) Kaiserslautern, Germany, as a senior lecturer. He served as a vice Chair of IEEE TCCN special interest group (SIG) "Cognitive Radio in 5G". He is the author of more than 30 papers in top international journals and conference proceedings, and has 27 patent applications in wireless communications, most of which have already been authorized in China, Europe, United States or Japan. He wrote a chapter "From OFDM to FBMC: Principles and Comparisons" for the book "Signal Processing for 5G: Algorithms and Implementations" (Wiley, 2016). His present research interests are in digital signal processing, multi-antenna technology, cooperative communications, 5G, and machine learning. 